\title{
Esquema para Redução de PAPR em Sistemas OFDM empregando as Técnicas SLM e Double WHT
}

\author{
Guilherme Pedro Aquino, Luciano Leonel Mendes e Tales Cleber Pimenta
}

\begin{abstract}
Resumo - OFDM é amplamente empregado em padrões que utilizam a tecnologia de Rádio Cognitivo. No entanto, a elevada PAPR do sinal OFDM é um desafio, pois os picos do sinal podem saturar o amplificador, resultando em emissão fora da faixa em possível interferência em usuários primários. Como isso não é tolerado, a alta PAPR pode impedir que Rádios Cognitivos entrem em operação. $O$ objetivo deste artigo é apresentar um esquema para reduzir a PAPR combinando o SLM e Double WHT com o OFDM. Além da redução da PAPR, este esquema melhora o desempenho em canais seletivos e aumenta a robustez à ICI.
\end{abstract}

\section{Palavras-Chave - OFDM, PAPR, WHT, SLM, Desempenho.}

Abstract - OFDM is widely used in Cognitive Radio systems. However, the high PAPR of the OFDM signal is a challenge because high amplitude values can drive the amplifier to saturation. Clipping introduces by the amplifier results on out-ofband emissions that can interfere in the performance of primary users on adjacent channels. Since this is not allowed in cognitive radio system, high PAPR can prevent cognitive radio from operating. The aim of this paper is to present a scheme to reduce PAPR using SLM and Double WHT techniques. This scheme also increases the performance over frequency-selective channels and the robustness against ICI.

\section{Keywords - OFDM, PAPR, WHT, SLM, Performance.}

\section{INTRODUÇÃO}

O advento de dispositivos portáteis com elevada capacidade de processamento, armazenamento e de geração de conteúdos está estimulando a demanda por maiores taxas de transmissão em redes móveis sem fio. A utilização oportunista do espectro de frequência vem sendo considerada uma solução interessante para atender esta crescente demanda, principalmente porque a alocação fixa do espectro de frequências resulta em uma subutilização deste recurso [1]. Para que o terminal de rádio possa utilizar o espectro de forma oportunista, é necessário que o mesmo tenha capacidade de analisar o ambiente espectral no qual se encontra e tomar a decisão de ocupar um dado canal livre, também chamado de white space, sem causar interferências nos usuários primários. Este dispositivo foi primeiramente proposto por Joseph Mitola III e recebeu o nome de Rádio Cognitivo (RC) [2].

Em uma rede de RCs, é fundamental garantir que a operação oportunista dos terminais não cause interferências nos usuários primários, que são aqueles que detêm o direito de uso do espectro. O sensoriamento espectral [3] [4] [5] possui um papel importante neste aspecto, pois é através desta técnica que os RCs determinam quais canais estão ocupados e quais canais podem ser usados de forma oportunista. Outra solução para evitar interferências em usuários primários consiste em utilizar base de dados com as informações de todos os

Guilherme Pedro Aquino e Luciano Leonel Mendes, Instituto Nacional de Telecomunicações (Inatel), Santa Rita do Sapucaí-MG, Brasil, E-mails: guilhemeaquino@inatel.br, luciano@inatel.br.

Tales Cleber Pimenta, Universidade Federal de Itajubá (UNIFEI), Itajubá-MG, Brasil, E-mail: tales@unifei.edu.br. transmissores da rede primária [6] [7]. Esta solução está sendo considerada para viabilizar a implantação de redes de RCs na faixa de UHF, sem causar interferência nas emissoras de televisão. Neste caso, antes de uma estação radiobase (ERB) de uma rede de RCs entrar em funcionamento, esta deve fornecer sua localização, área de cobertura pretendida, potência de transmissão, entre outros dados, para uma base de dados. Com estas informações, e tendo conhecimento dos dados referentes a todos os transmissores dos serviços primários na faixa de frequência de interesse, a base de dados retorna à ERB o canal no qual a mesma está autorizada a operar. Esta abordagem permite reduzir o custo dos terminais RCs, mas não é adequada para cenários onde usuários primários possam iniciar a transmissão de forma aleatória.

Outro fator importante para a operação do rádio cognitivo é a modulação empregada na camada física. De uma forma geral, redes de RCs devem atender às demandas de comunicação de dispositivos móveis, o que significa que o canal de comunicação apresenta múltiplos percursos e desvio de frequência Doppler. O OFDM (Orthogonal Frequency Division Multiplexing) [8] é amplamente empregado nos padrões de comunicação de elevada vazão, como WiMAX (Wireless Interoperability Microwave Access) [9], Wi-FI (Wireless Fidelity) [10], LTE (Long Term Evolution) [11], em função de sua robustez aos efeitos introduzidos pelos múltiplos percursos do canal de comunicação e simplicidade de implementação. Apesar de todas as vantagens apresentadas pelo OFDM, esta técnica apresenta uma característica especialmente crítica para RCs, que é a elevada PAPR (Peak to Average Power Ratio) [12]. Um sinal com elevada PAPR pode apresentar picos de amplitude diversas ordens de grandeza maiores do que seu desvio padrão. Esses picos de amplitude levam o amplificador de potência à saturação, resultando no ceifamento da forma de onda transmitida e, consequentemente, no aumento da emissão fora da faixa e ICI (Intra-carrier Interference). A emissão fora da faixa pode causar interferência em usuários primários e secundários que ocupam canais adjacentes, enquanto que a ICI resulta em aumento da taxa de erro de símbolo do usuário secundário.

Há diversos trabalhos na literatura que propõem esquemas para a redução da PAPR do sinal OFDM. Em [13] os autores propõem o uso de uma curva de compressão no transmissor e de uma curva de expansão no receptor para evitar a saturação do amplificador. No entanto, a natureza não-linear desta operação introduz ICI e não elimina completamente a emissão fora da faixa. Já em [14] os autores apresentam um esquema chamado de SLM (Selective Modulation) onde diferentes símbolos OFDM são gerados a partir do mesmo vetor de dados e aquele que apresenta a menor PAPR é selecionado para a transmissão. A geração de diferentes símbolos é realizada através da multiplicação do vetor de dados por $U$ diferentes sequências pseudo-aleatórias. Neste caso, o 
transmissor deve informar ao receptor a sequência utilizada na geração de cada um dos símbolos transmitidos. Esta informação deve ser devidamente protegida usando, por exemplo, códigos corretores de erro, pois caso esta seja recebida de forma equivocada haverá erro na recepção de todo o vetor de dados. Um grande desafio para a implementação desta técnica consiste na complexidade para a realização de $U$ IFFT (Inverse Fast Fourier Transform) no transmissor no intervalo de tempo de um símbolo OFDM.

Um esquema interessante para redução da PAPR, proposto em [15], consiste em utilizar a WHT (Walsh-Hadamard Transform) combinada com o OFDM para reduzir a PAPR. Esta solução também permite melhorar o desempenho em canais seletivos em frequência, pois cada subportadora passa a transmitir uma combinação linear de todos os símbolos do vetor de dados. Assim, caso uma subportadora sofra um desvanecimento mais severo não há perda de um símbolo em específico, mas há uma degeneração em todos os símbolos do vetor. A desvantagem desta técnica é que a correlação dos símbolos no domínio da frequência resulta em uma maior sensibilidade a ruídos impulsivos no domínio do tempo. Embora haja uma redução da PAPR e, consequentemente uma redução na probabilidade de ocorrência de ceifamento, a ocorrência de picos no sinal WHT-OFDM ainda pode levar o amplificador à saturação e a introdução do ceifamento, o que resulta em um patamar de erro de símbolo elevado [16]. Em [17] os autores apresentaram um esquema que permite reduzir a correlação dos símbolos de dados no domínio da frequência, reduzindo a sensibilidade ao ceifamento temporal, mas mantendo o ganho de desempenho proporcionado pela WHT em canais seletivos em frequência. No entanto, o esquema proposto não permite o controle da PAPR, cuja redução depende da combinação linear das componentes reais e imaginárias dos símbolos de dados e das matrizes de WalshHadamard utilizadas.

O objetivo deste artigo consiste em apresentar uma proposta para controle da PAPR combinando as técnicas SLM e Double WHT-OFDM, visando o controle da PAPR, além de analisar o desempenho da técnica proposta em comparação com outros esquemas de controle de PAPR. Para atingir estes objetivos o restante do artigo está organizado da seguinte forma: A Sessão II traz um breve resumo da técnica WHTOFDM, enquanto que a Sessão III traz os princípios do Double WHT-OFDM. Já a Sessão IV apresenta os conceitos do SLM e a Sessão V apresenta o esquema proposto neste artigo. Finalmente, a Seção VI traz as conclusões deste trabalho.

\section{PRINCÍPIOS DA TÉCNICA WHT-OFDM}

A técnica WHT-OFDM, cujo diagrama simplificado do transmissor e receptor é apresentado na Figura 1, consiste em aplicar a WHT no vetor de $N$ símbolos complexos de dados $\mathbf{C}^{T}=\left[c_{1}, c_{2}, \ldots, c_{N}\right]$ provenientes de um modulador $M$-QAM (Quadrature Amplitude Modulation), ou seja, o vetor de dados utilizados para a geração do símbolo OFDM é dado por

$$
\mathbf{S}=\frac{1}{\sqrt{N}} \mathbf{\Omega}_{\mathrm{N}} \mathbf{C},
$$

onde $N$ é o número de subportadoras empregadas no OFDM e

$$
\boldsymbol{\Omega}_{\mathrm{N}}=\left[\begin{array}{cc}
\boldsymbol{\Omega}_{\mathrm{N} / 2} & \boldsymbol{\Omega}_{\mathrm{N} / 2} \\
\boldsymbol{\Omega}_{\mathrm{N} / 2} & -\boldsymbol{\Omega}_{\mathrm{N} / 2}
\end{array}\right],
$$

é a matriz quadrada de Walsh-Hadamard de dimensão $N$, sendo $\boldsymbol{\Omega}_{1}=1$. O símbolo OFDM é gerado aplicando a IFFT no vetor proveniente da WHT, de forma que este é dado por

$$
s[n]=\frac{1}{\sqrt{N}} \sum_{k=0}^{N-1} S[k] \cdot e^{j \frac{2 \pi}{N} k n}, \quad n=0,1, \cdots, N-1,
$$

onde $n=0,1,2, \ldots, N$-1 é o índice no domínio do tempo e $k$ é o índice das subportadoras.

De acordo com (1) e (3) cada subportadora do símbolo OFDM transporta um combinação linear dos $N$ símbolos de dados, o que significa que caso uma dada subportadora sofra um desvanecimento severo, as informações disponíveis nas demais subportadoras pode ser suficiente para recuperar todos os dados, sem perda significativa de informação. Assim, obtém-se um ganho de desempenho em canais seletivos em frequência em função desta diversidade no domínio da frequência [16].

No receptor, o sinal proveniente do canal de comunicação r é aplicado ao bloco da FFT, resultado em

$$
R[k]=\frac{1}{\sqrt{N}} \sum_{n=0}^{N-1} r[n] \cdot e^{-j \frac{2 \pi}{N} k n}, \quad k=0,1, \cdots, N-1 .
$$

O vetor $\mathbf{R}$ é aplicado ao bloco da transformada inversa de Walsh-Hadamard, resultando no vetor de símbolos de dados estimados, dado por

$$
\hat{\mathbf{C}}=\frac{1}{\sqrt{N}} \mathbf{\Omega}_{\mathrm{N}} \mathbf{R},
$$

que são utilizados pelo demodulador $M$-QAM para estimar os bits transmitidos.
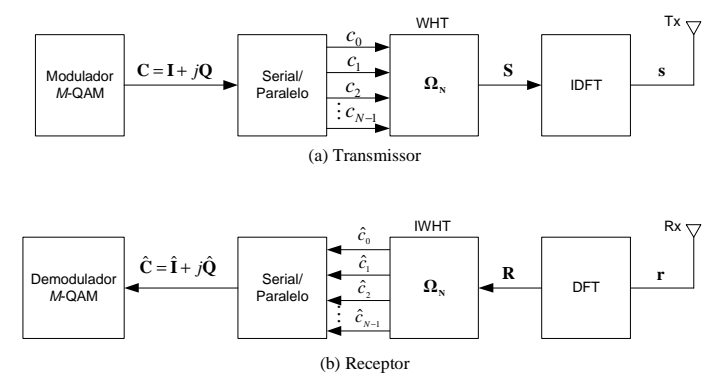

Figura 1. Diagrama em blocos do (a) transmissor e (b) receptor do esquema WHT-OFDM.

A Figura 2 apresenta os desempenhos teóricos dos sistemas OFDM convencional e WHT-OFDM em um canal seletivo em frequência [18]. Além disso, esta figura apresenta o desempenho simulado das duas técnicas quando o canal apresenta ceifamentos de pico. A Tabela 1 apresenta os parâmetros empregados nesta simulação, onde $\sigma_{s}$ é o desvio padrão da parte real do símbolo OFDM convencional. O canal utilizado é o Brasil A [19].

Tabela 1. Parâmetros empregados nas simulações.

\begin{tabular}{|l|c|}
\hline \multicolumn{1}{|c|}{ Parâmetro } & Valor \\
\hline Modulação & 16-QAM \\
\hline Número de subportadoras & 2048 \\
\hline Limiar de ceifamento & $2 \sigma_{s}$ \\
\hline
\end{tabular}

Conforme já mencionado, a transformada de WalshHadamard reduz a PAPR do símbolo OFDM e aumenta a robustez do OFDM em um canal seletivo em frequência. Contudo, em canal não-linear, o WHT-OFDM apresenta um patamar de erro de símbolo mais elevado do que o OFDM convencional, em função da maior sensibilidade do WHTOFDM ao ceifamento [16]. Logo, o WHT-OFDM é uma solução interessante apenas para canais lineares seletivos em frequência. 


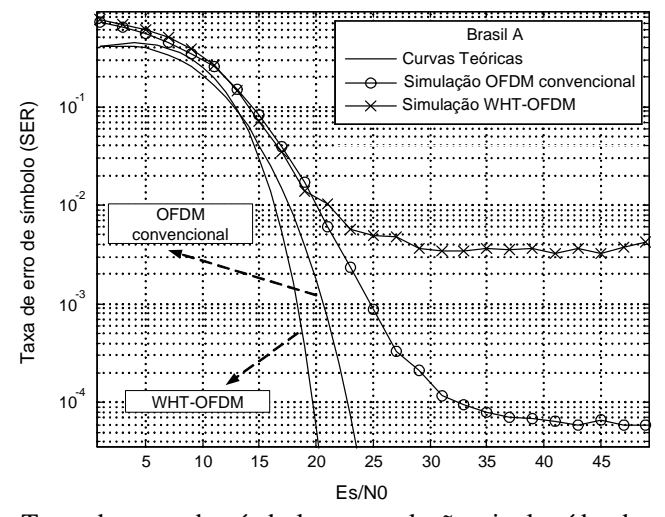

Figura 2. Taxa de erro de símbolos vs. relação sinal-ruído dos esquemas OFDM e WHT-OFDM no canal Brasil A não-linear [17].

\section{PRINCÍPIOS DA TÉCNICA DOUBLE WHT-OFDM}

O objetivo da técnica Double WHT-OFDM, cujo diagrama em blocos do transmissor e receptor é apresentado na Figura 3, é manter o ganho de desempenho frente a canais seletivos em frequência, sem o ônus de aumentar a sensibilidade do sistema em caso de ceifamento por parte do amplificador de potência. Desta forma, o Double WHT-OFDM permite utilizar a transformada de Walsh-Hadamard em canais não lineares. Neste esquema, duas diferentes matrizes de Walsh-Hadamard são utilizadas para a geração do vetor a ser aplicado na IFFT. A primeira matriz, $\mathbf{\Omega}_{\mathrm{N}}^{\mathrm{xy}}$, é obtida através da permutação das colunas $x$ e $y$ da matriz de Walsh-Hadamard original e empregada para realizar a WHT da parte real de $\mathbf{C}$, enquanto que a segunda matriz, representada por $\mathbf{\Omega}_{\mathrm{N}}^{\mathrm{kl}}$, é obtida através da permutação das colunas $l$ e $k$ da matriz de Walsh-Hadamard e é empregada para realizar a WHT da parte imaginária de $\mathbf{C}$.
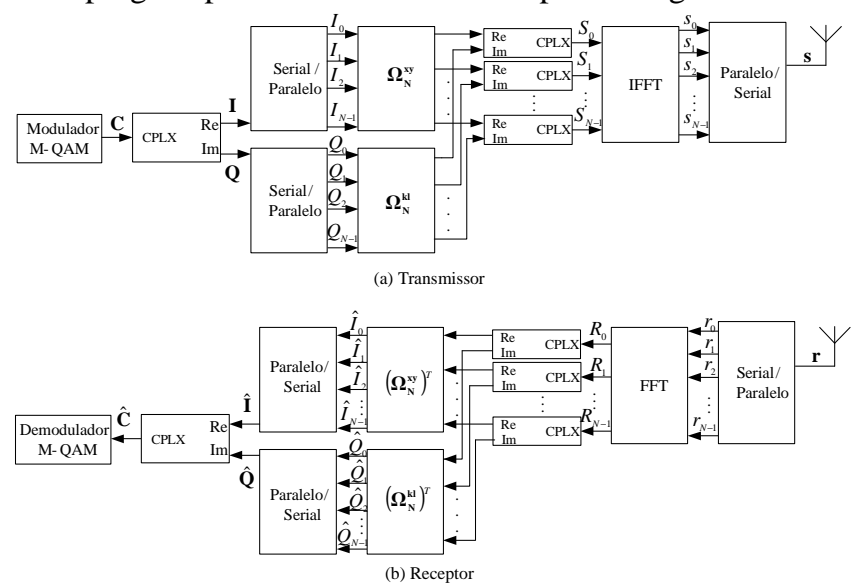

Figura 3. Diagramas em blocos do (a) transmissor e (b) receptor do esquema Double WHT-OFDM.

No receptor, o sinal proveniente do canal de comunicação é digitalizado e paralelizado e entregue para a FFT. A parte real do vetor na saída da FFT sofre a transformada inversa de Walsh-Hadamard com a matriz $\boldsymbol{\Omega}_{\mathrm{N}}^{\mathrm{xy}}$, enquanto que a parte imaginária deste vetor sofre a transformada inversa de WalshHadamard empregando a matriz $\mathbf{\Omega}_{\mathrm{N}}^{\mathrm{kl}}$. É importante notar que pelo fato das colunas da matriz de Walsh-Hadamard terem sido permutadas, a operação inversa agora difere da transformada direta, uma vez que na IWHT deve-se utilizar a transposta da matriz empregada na WHT. Assim, tem-se que

$$
\hat{\mathbf{C}}=\frac{1}{\sqrt{N}}\left\{\left(\mathbf{\Omega}_{\mathrm{N}}^{\mathbf{x y}}\right)^{T} \mathfrak{R} e(\mathbf{R})+j \cdot\left(\mathbf{\Omega}_{\mathrm{N}}^{\mathbf{k l}}\right)^{T} \mathfrak{I} m(\mathbf{R})\right\} .
$$

Após o processo da transformada inversa de WalshHadamard, os símbolos de dados recebidos são entregues para o demodulador $M$-QAM para estimar os bits recebidos. A Figura 4 apresenta a taxa de erro de símbolo do OFDM, WHT-OFDM e Double WHT-OFDM no canal Brasil E [19], onde é possível observar que o Double WHT-OFDM não sofre uma perda significativa em função do ceifamento, quando comparado com o OFDM.

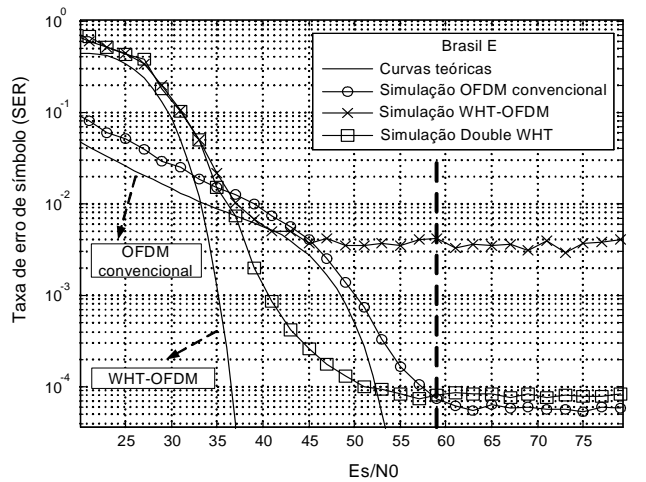

Figura 4. Taxa de erro de símbolos vs. relação sinal-ruído dos esquemas OFDM, WHT-OFDM e Double WHT-OFDM no canal Brasil E não-linear [17].

Assim, pode-se concluir que o Double WHT-OFDM apresenta maior robustez frente ao ceifamento de pico, preservando as vantagens da utilização da transformada de Walsh-Hadamard em canais seletivos em frequência.

\section{PRINCÍPIOS DA TÉCNCIA SLM}

Conforme já mencionado, no esquema SLM são gerados $U$ símbolos OFDM distintos contendo a mesma informação. Para obter as diferentes versões, o vetor $\mathbf{C}$ é multiplicado por cada coluna da matriz de sequências pseudo-alatórias dada por

$$
\mathbf{P}=\left[\begin{array}{ccccc}
p_{11} & p_{12} & p_{13} & \cdots & p_{1 U} \\
p_{21} & p_{22} & p_{23} & \cdots & p_{2 U} \\
& \vdots & \vdots & \ddots & \vdots \\
p_{N 1} & p_{N 2} & p_{N 3} & \cdots & p_{N U}
\end{array}\right]
$$

Um seletor escolhe qual versão dentre as $U$ disponíveis possui a menor PAPR $\left(\mathbf{s}_{\text {slm }}\right)$ e também informa ao receptor, através de subportadoras de controle, o índice da sequência que foi utilizada na geração deste símbolo $\left(I P_{u}\right)$. No receptor, esta informação será empregada para selecionar a sequência pseudo-aleatória correta para a decodificação da informação. A Figura 5 apresenta o diagrama em blocos do esquema SLM. A grande desvantagem do SLM com relação ao WHT-OFDM e Double WHT-OFDM é que esta solução não traz ganho de desempenho em canais seletivos em frequência. Em compensação, o SLM resulta em uma maior redução da PAPR quando comparado com o WHT-OFDM e Double WHTOFDM [16].

O Double WHT-OFDM é uma solução interessante para aumentar o desempenho de sistemas OFDM em canais seletivos em frequência, sem o ônus de resultar em um elevado patamar de erro de símbolo caso o amplificador ceife os picos do sinal. No entanto, este esquema não permite o controle da PAPR. Em contrapartida, a técnica SLM permite uma redução significativa da PAPR, mas sem o benefício de melhorar o desempenho do sistema em canais seletivos em frequência.

\section{SLM-DWHT-OFDM}

O esquema proposto neste artigo visa combinar a técnica SLM com o Double WHT-OFDM com o objetivo de controlar a PAPR do sinal transmitido e, assim, minimizar a 
probabilidade de ceifamento e, consequentemente, a emissão fora da faixa, mantendo o ganho em canais seletivos em frequência proporcionado pela transformada de WalshHadamard. O diagrama em blocos do transmissor deste esquema, denominado de SLM-DWHT-OFDM, é apresentado na Figura 8, enquanto que a Figura 9 apresenta o diagrama em blocos do receptor.

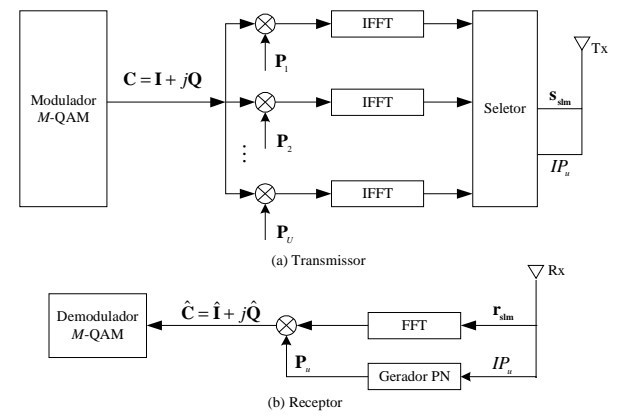

Figura 5. Diagramas em blocos do (a) transmissor e (b) receptor do esquema SLM.

A técnica SLM-DWHT-OFDM utiliza várias combinações de matrizes de Walsh-Hadamard diferentes para diminuir a PAPR do sinal transmitido $\mathbf{S}_{\mathbf{D}}$. No início do processo são escolhidas $U$ matrizes de Walsh-Hadamard diferentes, que serão empregadas nas transformadas direta e inversa de Walsh-Hadamard da parte real e da parte imaginária de C. O vetor $\mathbf{S}$ proveniente da WHT é aplicado no bloco que computa a IFFT, gerando o sinal s. Um comparador calcula a PAPR deste sinal (s) e compara com o valor da PAPR máxima desejada. Caso a PAPR do sinal s seja menor do que a PAPR máxima, então este é transmitido juntamente com a informação dos índices das matrizes utilizadas para a sua geração, $I \Omega_{N}^{x y}$ e $I \Omega_{N}^{k l}$. Caso a PAPR seja maior do que a PAPR máxima, então um novo par de matrizes de Walsh-Hadamard é selecionado para a geração de um novo sinal s. Este processo se repete até que seja obtido um símbolo com PAPR menor do que a PAPR máxima ou até que todas as combinações possíveis com as matrizes selecionadas sejam atingidas. No segundo caso, o símbolo com menor PAPR dentre todos os possíveis é enviado.

No receptor, a informação dos índices das matrizes empregadas para gerar o símbolo no transmissor é decodificada das subportadoras de controle e utilizada no processo da transformada inversa de Walsh-Hadamard. A partir deste ponto, o receptor do SLM-DWHT-OFDM opera exatamente do mesmo modo que o receptor do esquema Double WHT-OFDM.

É interessante observar que: (i) quanto maior for o valor da $P A P R_{\text {max }}$, maior será a probabilidade de se atender a este critério com as $U^{2}$ combinações de matrizes de WalshHadamard e; (ii) se o valor de $P A P R_{\max }$ for muito baixo é possível que este critério não seja atingido mesmo que o número de matrizes empregadas no processo seja elevado. Com o aumento do número de matrizes há um aumento significativo da complexidade computacional para a implementação do esquema e menor eficiência espectral, em função da necessidade de transmitir uma maior quantidade de bits para informar os índices das matrizes para o receptor. Outro ponto importante é que a informação referente aos índices das matrizes empregadas pelo transmissor para a geração do sinal transmitido deve ser adequadamente protegida, uma vez que um erro introduzido nesta informação resulta na perda de todos os símbolos de dados do respectivo símbolo $\mathbf{s}$.

A Figura 6 apresenta a probabilidade da PAPR assumir um valor, em escala linear, maior do que um dado limiar, obtida para os esquemas apresentados neste artigo, considerando as mesmas características listadas na Tabela 1.

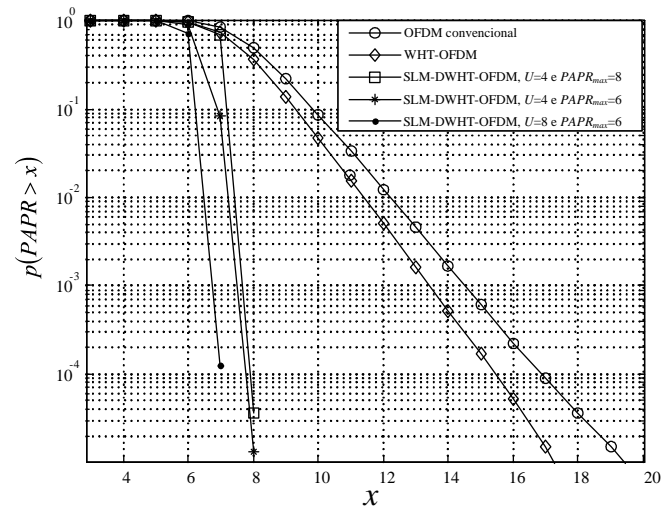

Figura 6. Probabilidade da PAPR ser maior do que um dado limiar para o OFDM, WHT-OFDM e SLM-DWHT-OFDM.

Observando os resultados apresentados na Figura 6 é possível concluir que o esquema proposto neste artigo é capaz de reduzir consideravelmente a PAPR do sinal transmitido, quando comparado com o WHT-OFDM. Além disso, é possível observar que com quatro matrizes de WalshHadamard é possível atender ao critério de $P A P R_{\max }=8$, ao passo que a restrição de $P A P R_{\max }=6$ não é atendida nem mesmo quando $U=8$, embora neste caso haja uma redução clara da probabilidade da PAPR ser maior do que 6 quando comparado com o resultado obtido com $U=4$.

A Figura 7 compara o desempenho em termos de taxa de erro de símbolo do esquema proposto com o OFDM e WHTOFDM, considerando $P A P R_{\max }=6$.

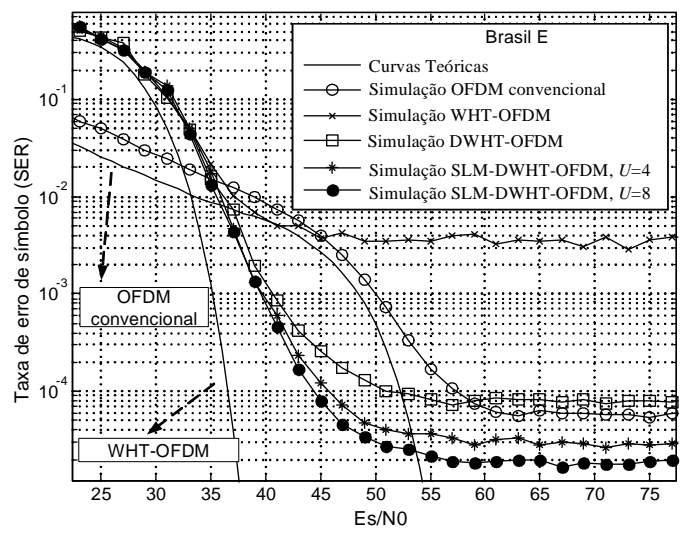

Figura 7. Taxa de erro de símbolos vs. relação sinal-ruído dos esquemas OFDM, WHT-OFDM e SLM-DWHT-OFDM com $P A P R_{\max }=6$ no canal Brasil E não-linear.

Conforme é possível observar na Figura 7, o desempenho do SLM-DWHT-OFDM supera o OFDM e WHT-OFDM. Para a faixa de relação sinal-ruído entre $35 \mathrm{~dB}$ e $60 \mathrm{~dB}$, onde a ação seletiva do canal é mais significativa do que o efeito de ceifamento no OFDM, é possível observar que o uso da transformada de Walsh-Hadamard, no esquema proposto neste artigo, resulta em um expressivo ganho de desempenho. Já para elevados valores de relação sinal-ruído, onde o ceifamento do sinal se torna o fator mais limitante, o SLMWHT-OFDM atinge um patamar de erro inferior ao OFDM, o que mostra que o uso do SLM resulta em um melhor desempenho do sistema frente aos efeitos não lineares do canal de comunicação. 


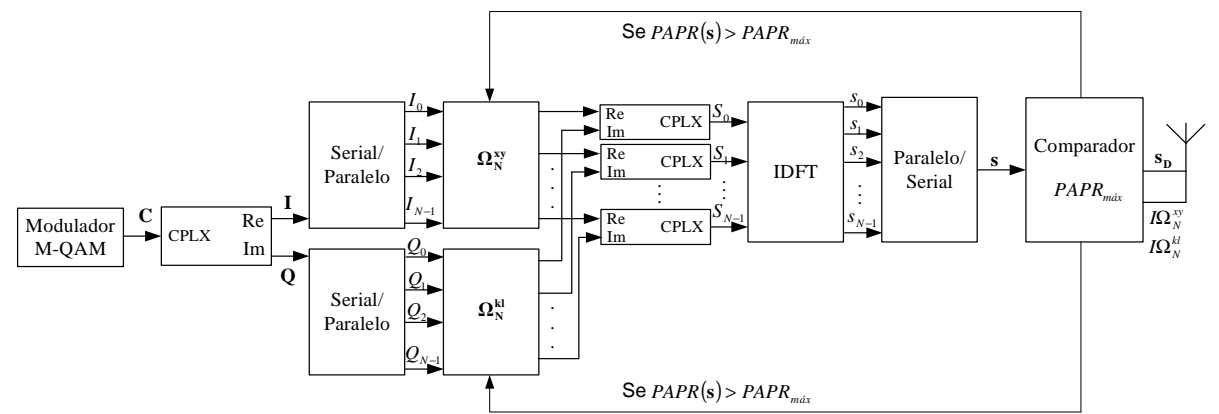

Figura 8. Diagrama em blocos do transmissor do esquema SLM-DWHT-OFDM.

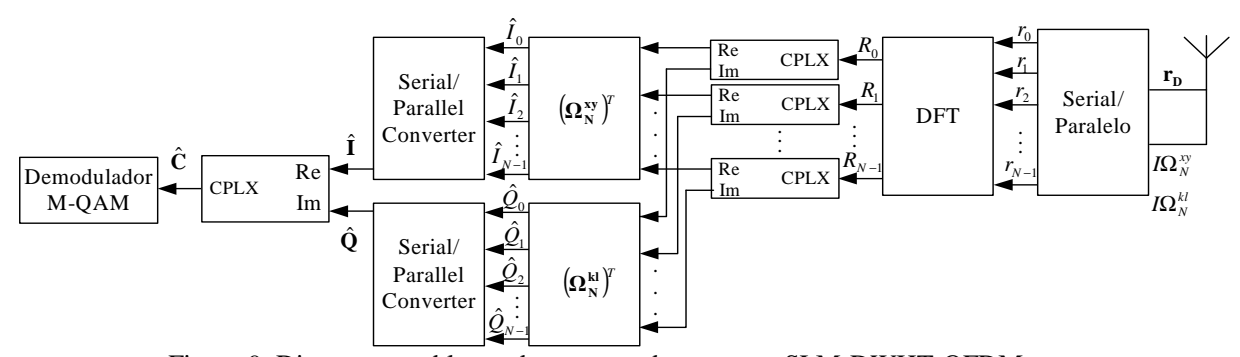

Figura 9. Diagrama em blocos do receptor do esquema SLM-DWHT-OFDM.

\section{CONCLUSÕES}

A elevada PAPR do sinal OFDM é um limitante importante para a utilização desta tecnologia na camada física de RCs, uma vez que os elevados picos do sinal podem saturar os amplificadores de potência, resultando em emissões fora da faixa e aumento da probabilidade de erro de símbolo.

A proposta apresentada neste trabalho agrega duas técnicas de controle de PAPR que trazem benefícios complementares para a operação em canais seletivos em frequência. Enquanto que a técnica SLM permite reduzir o valor da PAPR, a Double WHT aumenta a robustez em canais seletivos em frequência e reduz a sensibilidade do sinal aos eventuais ceifamentos introduzidos pelo amplificador de potência do transmissor.

A complexidade de implementação do esquema proposto depende principalmente do número de matrizes que podem ser usadas no esquema. As simulações mostram que um número maior de matrizes reduz tanto o valor da PAPR quanto a taxa de erro, ao custo de uma maior complexidade computacional e menor eficiência espectral.

\section{AGRADECIMENTOS}

Os autores gostariam de agradecer ao Instituto Nacional de Telecomunicações pelo apoio e suporte para o desenvolvimento deste trabalho.

\section{REFERENNCIAS}

[1] K. Harrison, S. M. Mishra, e A. Sahai, "How Much White-Space Capacity Is There?", in Proceedings of IEEE Symposium on New Frontiers in Dynamic Spectrum, Singapore, 2010, p. 1-10.

[2] J. Mitola, "Cognitive radio for flexible mobile multimedia communications", in Proceeding of IEEE International Workshop on Mobile Multimedia Communications, San Diego, CA, USA, 1999, p. $3-10$.

[3] A. Ghasemi e E. S. Sousa, "Spectrum sensing in cognitive radio networks: requirements, challenges and design trade-offs", Ieee Commun. Mag., vol. 46, $\mathrm{n}^{\circ}$ 4, p. 32-39, abr. 2008.

[4] Y. Zeng, Y.-C. Liang, A. T. Hoang, e R. Zhang, "A Review on Spectrum Sensing for Cognitive Radio: Challenges and Solutions", Eurasip J. Adv. Signal Process., vol. 2010, p. 1-16, 2010.

[5] E. Axell, G. Leus, E. Larsson, e H. Poor, "Spectrum Sensing for Cognitive Radio: State-of-the-Art and Recent Advances", Ieee Signal Process. Mag., vol. 29, nº 3, p. 101-116, maio 2012.

[6] S. Shellhammer, A. Sadek, e W. Zhang, "Technical Challenges for Cognitive Radio in the TV White Space Spectrum", in Proccedings of
Information Theory and Applications Workshop, San Diego, CA, USA, 2009, p. 323-333.

[7] J. Paavola, "Operational Challenges for Emerging Cognitive Radio Technologies - Wireless Devices Utilizing TV White Spaces", in Proceedings of 10th FRUCT Conference, Tampere, Finland, 2011, p. $105-111$.

[8] A. R. S. Bahai e B. R. Saltzberg, Multi-carrier digital communications theory and applications of OFDM. New York: Kluwer Academic/Plenum, 1999.

[9] M. A. Hasan, "Performance Evaluation of WiMAX/IEEE 802.16 OFDM Physical Layer", Helsinki University of Technology, Finland, 2007.

[10] Radio Electronics, "Wi-Fi: Standards and Applications Tutorial", IEEE 802.11 Standards Tutorial, 09-fev-2012. [Online]. Available at: http://www.radio-electronics.com/info/wireless/wi-fi/ieee-802-11standards-tutorial.php.

[11] Mobile Broadband Including WiMAX and LTE. Boston, MA: Springer Science Business Media, LLC, 2009.

[12] Ahmad Bahai, Manonnet Singh, Andrea Goldsmith, e Burton Saltzberg, "A New Approach for Evaluating Clipping Distortion in Multicarrier Systems", Ieee J. Sel. Areas Commun., vol. 20, no 5, nº 5, p. 1037-1046, jun. 2002.

[13] T. Jiang, Y. Yang, e Y.-H. Song, "Companding Technique for PAPR Reduction in OFDM Systems Based on An Exponential Function”, in Proceeding of Globecom 2005, Saint Louis, USA, 2005.

[14] H.-S. Joo, S.-J. Heo, H.-B. Jeon, J.-S. No, e D.-J. Shin, "A New Blind SLM Scheme with Low Complexity of OFDM Signals", in Proceedings of Vehicular Technology Conference Fall, Anchorage, AK, USA, 2009, p. 1-5.

[15] Myonghee Park, Heeyoung Jun, Jaehee Cho, Namshin Cho, Daesik Hong, e Changeun Kang, "PAPR reduction in OFDM transmission using Hadamard transform", apresentado em IEEE International Conference on Communications, New Orleans, LA, USA, 2011, p. 430-433.

[16] G. Aquino, L. Mendes, e L. Resende, "Melhoria da técnica de redução da PAPR baseada na transformada de Walsh-Hadamard (WHTOFDM)", in Anais do SBrT 2012, Brasília, Brasil, 2012.

[17] L. Mendes, G. Aquino, e L. Resende, "Double Walsh-Hadamard Transform OFDM System", in Proceedings of International Workshop on Telecommunications, Santa Rita do Sapucaí, BR, 2013.

[18] L. Mendes e R. Baldini Filho, "Performance of WH-STC-OFDM in Mobile Frequency Selective Channel", in Proceedings of the ITS 2010, Manaus, AM, Brazil, 2010.

[19] G. Gomes e D. Guimarães, "Caracterização do canal para o MISBTVD”, Rev. Telecomunicações, vol. 9, n ${ }^{\circ}$ 1, p. 32-33, 2006. 